\title{
An Overview of Autonomous Underwater Vehicle Research and Testbed at PeRL
}

Hunter C. Brown, Ayoung Kim, Ryan M. Eustice

University of Michigan

\section{ABSTRACT}

This article provides a general overview of the autonomous underwater vehicle (AUV) research thrusts being pursued within the Perceptual Robotics Laboratory (PeRL) at the University of Michigan. Founded in 2007, PeRL's research centers on improving AUV autonomy via algorithmic advancements in environmentally-based perceptual feedback for real-time mapping, navigation, and control. Our three major research areas are: (1) real-time visual simultaneous localization and mapping (SLAM); (2) cooperative multi-vehicle navigation; and (3) perception-driven control. Pursuant to these research objectives PeRL has developed a new multi-AUV SLAM testbed based upon a modified Ocean-Server Iver2 AUV platform. PeRL upgraded the vehicles with additional navigation and perceptual sensors for underwater SLAM research. In this article we detail our testbed development, provide an overview of our major research thrusts, and put into context how our modified AUV testbed enables experimental real-world validation of these algorithms.

\section{Keywords: AUVs, SLAM, navigation, mapping, testbed}




\section{INTRODUCTION}

The Perceptual Robotics Laboratory (PeRL) at the University of Michigan (UMich) is actively involved in three major research efforts: real-time vision-based simultaneous localization and mapping (SLAM), heterogeneous multi-vehicle cooperative navigation, and perception-driven control. To test and experimentally validate these algorithms, we have developed a new multi-AUV testbed based upon a modified Ocean-Server Iver2 commercial AUV platform. This new AUV testbed provides a simple manportable platform for real-world experimental validation, and serves as a dedicated engineering testbed for proof-of-concept algorithmic implementations. In this article, we report on our developments in this area and provide an overview of this new experimental facility.

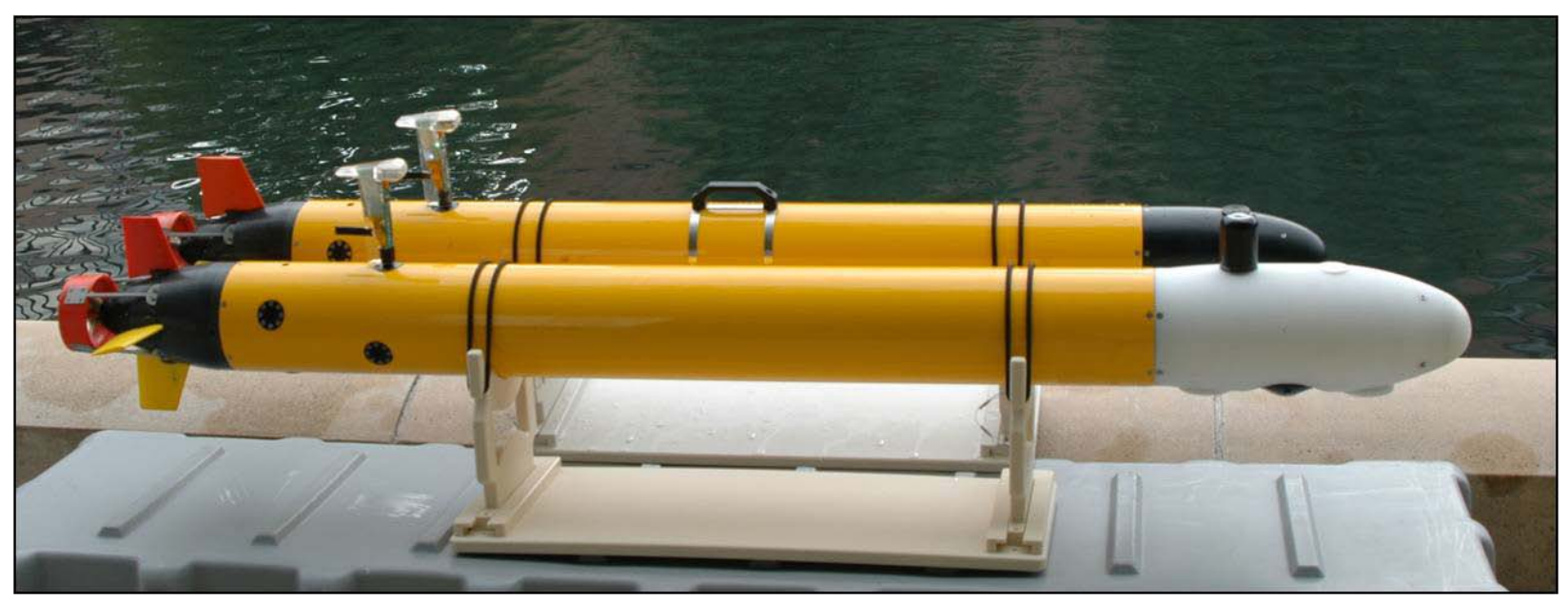

Figure 1. PeRL's multi-AUV testbed is based upon a modified Ocean-Server Iver2 AUV platform. Shown in the foreground is a modified vehicle displaying a new nose cone designed and fabricated by PeRL. For comparison, shown in the background is a stock vehicle displaying the original nose cone.

\subsection{Overview of Underwater Navigation}

One of the major limitations in the field of underwater robotics is the lack of radio-frequency transmission modes. The opacity of water to electromagnetic waves precludes the use of the Global Positioning System (GPS) as well as high-speed underwater radio communication (Stewart, 1991). Hence, communication and navigation underwater must rely upon other means. Kinsey et al (2006) 
provide an overview of the current state-of-the-art in underwater vehicle navigation, of which we briefly summarize here.

\subsubsection{Conventional Underwater Navigation Systems}

Two broad categories of underwater navigation methods exist for localizing vehicles and instruments: absolute positioning and relative dead-reckoning. The traditional long-baseline (LBL) method of underwater positioning estimates absolute position by measuring time-of-flight ranges to fixed beacons (Hunt, 1974; Milne, 1983). The precision of this estimate is bounded, and the accuracy is determined by system biases. The range of this solution is limited to a few kilometers in the best acoustic conditions and the positioning resolution is on the order of 1 meter. The slow update-rate of LBL is constrained by the acoustic travel times - typically updating every few seconds. In contrast to slow, coarse, but absolute LBL positioning, a Doppler velocity log (DVL) or inertial navigation system (INS) instead estimates distance traveled to infer position. Dead-reckoning is fast $(\sim 10 \mathrm{~Hz})$ and delivers fine resolution $(\sim 1 \mathrm{~cm})$, but the precision of this relative measurement is unbounded, growing monotonically with time. This makes it difficult to return to a known location or to relate measurements globally to one another.

\subsubsection{Underwater SLAM}

Over the past decade, a significant research effort within the terrestrial mobile robotics community has been to develop environmentally-based navigation algorithms that eliminate the need for additional infrastructure and bound position error growth to the size of the environment - a key prerequisite for truly autonomous navigation. The goal of this work has been to exploit the perceptual sensing capabilities of robots to correct for accumulated odometric error by localizing the robot with respect to landmarks in the environment (Bailey, 2006; Durrant-Whyte, 2006).

One of the major challenges of the SLAM problem is (i) defining fixed features from raw sensor data and

(ii) establishing measurement to feature correspondence [i.e., the problem of data association (Neira, 
2001)]. Both of these tasks can be nontrivial - especially in an unstructured underwater environment. In man-made environments, typically composed of planes, lines and corners primitives, point features can be more easily defined; however, complex underwater environments pose a more challenging task for feature extraction and matching.

One approach to curbing the challenges of defining fixed features from raw sensor data is to seed the environment with artificial landmarks that are easily recognizable. For example, one practical application of range-only underwater SLAM has been to localize an AUV in an acoustic beacon network that has a priori unknown geometry (Newman 2003; Olson 2006). In this scenario, the beacon geometry is learned online by the SLAM algorithm - eliminating the need for having to conduct an initial survey calibration of the acoustic beacon network by a surface ship. Moreover, this paradigm can easily be extended to a scenario where the AUV self-deploys the acoustic beacons in situ over the survey site.

More recently, progress has been made in applying SLAM in an a priori unknown underwater environment without the aid of artificial landmarks. In particular, one SLAM methodology that has seen recent success in the benthic realm is to apply a pose-graph scan-matching approach (Eustice 2006a; Fleischer, 2000). Pose-graph SLAM approaches do not require an explicit representation of features and instead use a data-driven approach based upon extracting relative-pose constraints from raw sensor data. The main idea behind this methodology is that registering overlapping perceptual data, for example optical imagery as reported in (Eustice, 2006a, 2006b) or sonar bathymetry as reported in (Roman, 2005), introduces spatial drift-free edge constraints into the pose-graph. These spatial constraints effectively allow the robot to close-the-loop when revisiting a previously visited place, thereby resetting any accumulated dead-reckoning error.

\subsection{Overview of PeRL's Research Thrusts}


PeRL's three major research areas are: (1) real-time visual simultaneous localization and mapping; (2) cooperative multi-vehicle navigation; and (3) perception-driven control. In this section we provide an overview of our lab's research thrusts as they pertain to AUV algorithms.

\subsubsection{Real-Time Visual SLAM}

The first of the three PeRL research domains, real-time vision-based SLAM algorithms, has direct application to areas such as autonomous underwater ship-hull inspection (Eustice, 2008a; Kim, 2009) and deep-sea archaeological missions (Ballard, 2008; Foley, 2009).

Present day means for ship hull and port security inspection require either putting divers in the water or piloting a remotely operated vehicle (ROV) over the area of interest - both of these methods are manpower intensive and generally cannot quantitatively guarantee $100 \%$ survey coverage. Automating this task; however, is challenging and compounded by the fact that areas around ships in berth are severely confined, cluttered, and complex sensing environments (e.g., acoustically, optically, magnetically). Current tethered robotic inspection systems present issues of snagging, maneuver degradation, and tether management, all of which make maneuvering around the ship at pier difficult. Moreover, current robotic inspection methods require human in-the-loop intervention for both sensory interpretation and control (e.g., ROV piloting). Navigation feedback in these scenarios is typically performed using acoustic transponder time-of-flight ranging (Smith, 1997). This necessitates setup and calibration of the acoustic-beacon infrastructure, and therefore vitiates our ability to rapidly and repeatably inspect multiple underwater structures.

Similarly, deep-sea archaeology also requires high-performance navigation (Ballard, 2008; Foley, 2009). Optical imagery, bathymetric sonar, and environmental measurements are all products of interest to the archeologist. The data may be collected over multiple missions or even multiple field seasons, and it is 
the precision of the navigation that makes it possible to transform these measurements into coregistered maps.

To combat the aforementioned navigation limitations (i.e., infrastructure-based and unbounded error growth), PeRL has been developing a camera-based navigation system that uses vehicle-collected imagery of the hull (port/hull-inspection) or seafloor (underwater archeology) to extract measurements of vehicle motion. These camera-derived spatial measurements are fused with the onboard deadreckoned data to produce a bounded-error navigation estimate (Figure 2).

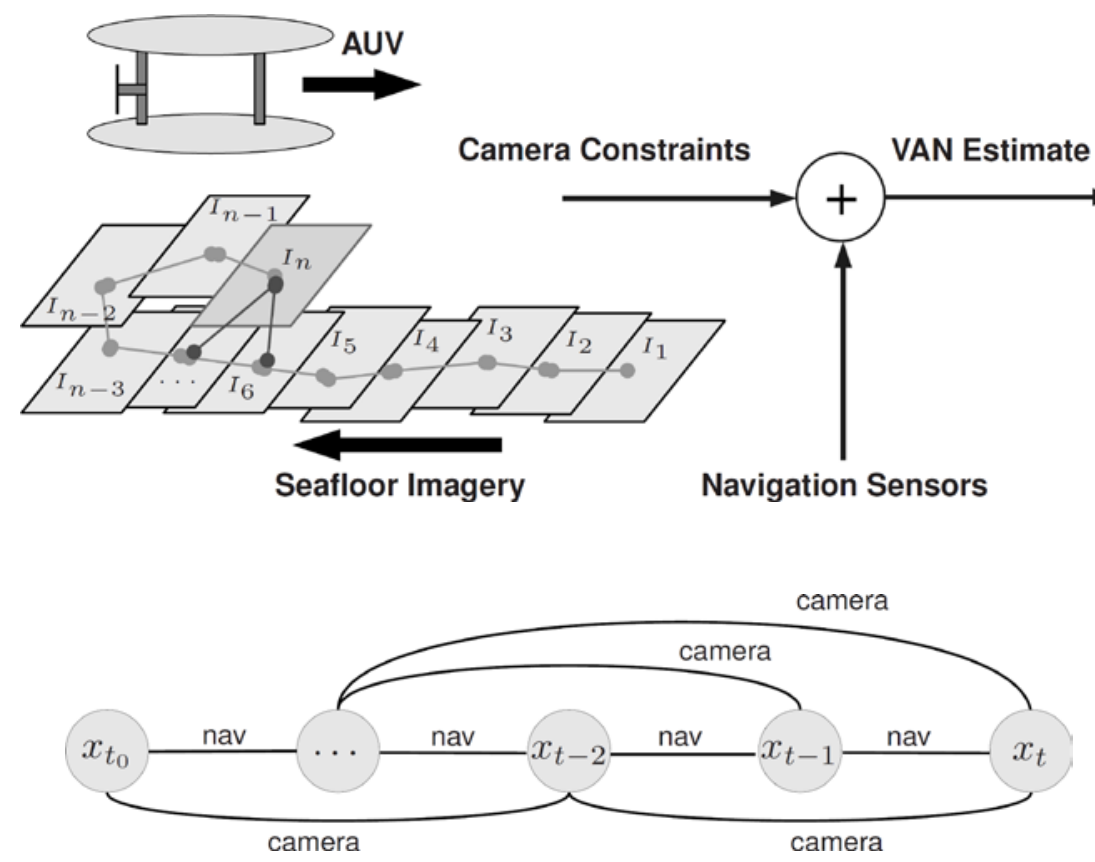

Figure 2. The foundation of visually augmented navigation (Eustice, 2008a) is the fusion of "zero-drift" camera measurements with dead-reckoned vehicle navigation data to produce a bounded error position estimate. These constraints are fused with onboard navigation sensor data in a view-based stochastic map framework; the model is comprised of a pose-graph where the nodes correspond to historical robot poses and the edges represent either navigation or camera constraints.

In essence, the AUV builds a digital map of the seafloor or hull by registering overlapping digital-still images (both along-track and cross-track imagery). Images that are successfully registered produce a relative measurement of the vehicle's attitude (heading, pitch, and roll) and translational ( $x, y, z)$ displacement. When fused with the onboard navigation data from a bottom-lock DVL, the result is a navigation system whose error is commensurate or much better than $L B L$, but which is infrastructure 
free. The significant advantage of this navigation paradigm is that it is in situ. For archeological surveys, this means that the AUV can be more easily deployed for exploratory surveys to investigate target ship wreck sites - without having to invest significant ship time in deploying a beacon-network to obtain precision navigation; and for hull inspection surveys, no additional acoustic beacon-infrastructure is required for precise global localization along the hull. In layman's terms, these algorithms allow the AUV to navigate much like a human does, by visually navigating with respect to the seafloor environment.

A useful and important by-product of this navigation methodology is that the overlapping registered imagery can be used to construct an optically-derived bathymetry map (Figure 3). This map can be used to construct a quantitatively accurate three-dimensional photomosaic by back-projecting the imagery over the optical bathymetry map. Figure 3 displays the result of applying this technology to the fourthcentry B.C. Chios classical ancient ship wreck site (Foley, 2009). In particular, Figure 3(a) shows the optically derived bathymetry map for a $15 \mathrm{~m}$ by $45 \mathrm{~m}$ swath centered overtop the wreck site. The optical bathymetry is generated from a 3D triangulated point cloud derived from pixel correspondences. In Figure 3(b), we display a quantitatively accurate three-dimensional photomosaic obtained by backprojecting the imagery onto the gridded surface. It should be emphasized that this result is fully automatic and metrically quantitative, in other words, measurements of object size and geometric relationships can be derived. While this technology is still very much in the active research stage, its current and future value for in situ, rapid, quantitative documentation of marine archeological wreck sites or ship hull inspection cannot be overstated. 


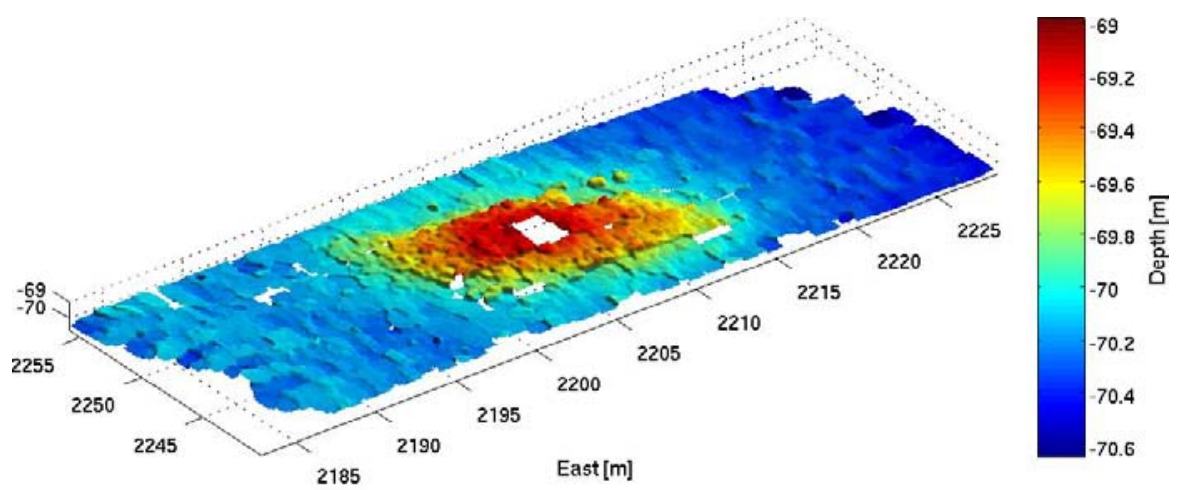

North $[\mathrm{m}]$

(a)

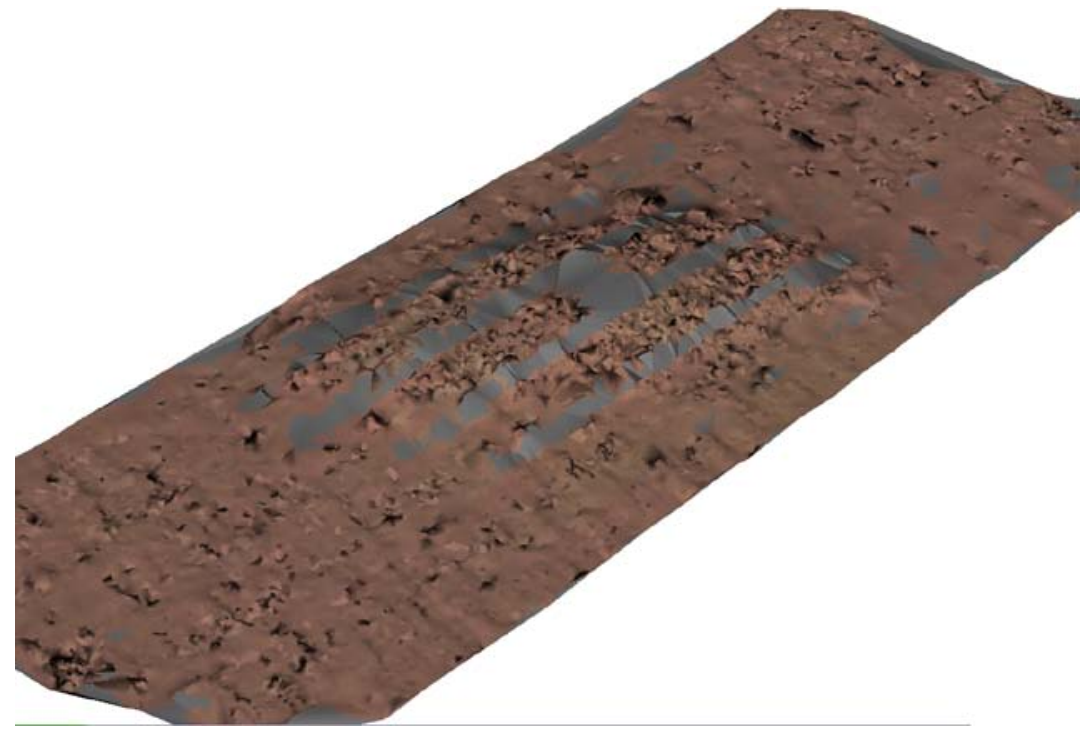

(b)

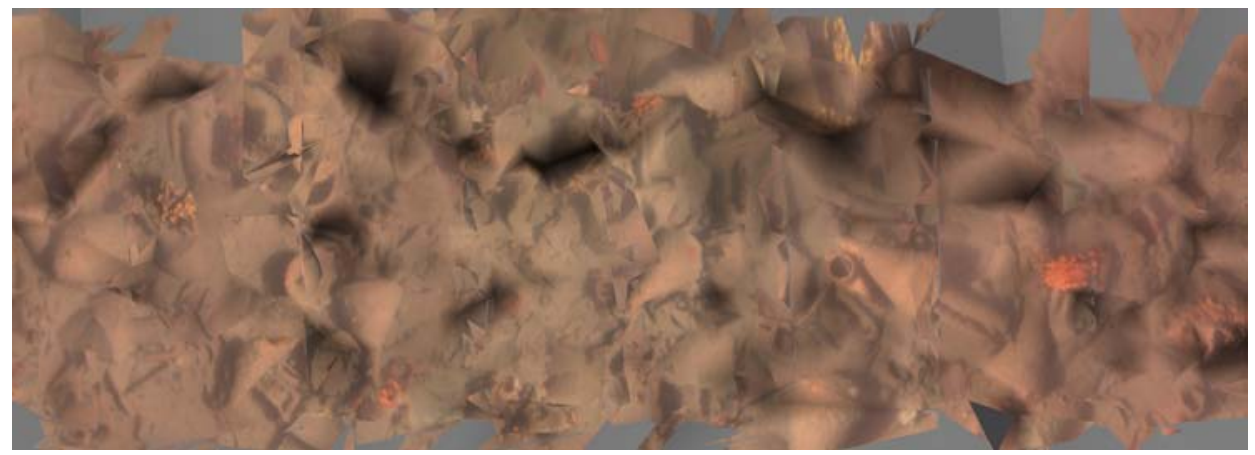

(c)

Figure 3. A by-product of camera-based AUV navigation is the ability to produce optically-derived bathymetry. Depicted are VAN-derived bathymetric maps from the Chios 2005 ship wreck survey (Foley, 2009). (a) Triangulated 3D point cloud from registered imagery. The point cloud is gridded to $5 \mathrm{~cm}$ to product an optically-derived bathymetry map. (b) A quantitatively correct three-dimensional mosaic generated by back-projecting the imagery onto the optical bathymetry map. The gray areas correspond to regions of no image overlap. (c) A zoomed overhead view shows amphorae pile detail in the center mound of the $3 d$ photomosaic. 


\subsubsection{Cooperative Navigation}

In addition to real-time visual SLAM, PeRL is working toward cooperative multi-vehicle missions for large-area survey. Multi-vehicle cooperative navigation offers promise of efficient exploration by groups of mobile robots working together to pool their mapping capability. Most prior research in the SLAM community has focused on the case of single-agent mapping and exploration. While these techniques can often be extended to a centralized multi-agent framework (Walter, 2004) (provided that there are no communication bandwidth restrictions), the extension of single-agent techniques to a decentralized multi-vehicle SLAM framework is often neither obvious nor appropriate. Much of the previous research in the area of distributed multi-vehicle SLAM has focused primarily on terrestrial (i.e., land and aerial) applications (Bourgault, 2004; Ong, 2006; Rekleitis, 2003; Ridley, 2002; Williams, 2002). There, highbandwidth radio communication is possible; however, underwater communication bandwidth is distinctly limited from that on land (Partan, 2006).

It requires on the order of 100 times more power to transmit than it does to receive underwater, which makes acoustic transmission and reception asymmetrical for medium access (MAC) schemes (Partan, 2006). Half duplex time division multiple access (TDMA) networks are usual, with typical acousticmodem data rates ranging from $5 \mathrm{kbits} / \mathrm{s}$ at a range of $2 \mathrm{~km}$ (considered a high rate), to as little as 80 bits/s (a low rate). The low acoustic data rates are not simply a limitation of current technology - the theoretical performance limit for underwater acoustic communications is $40 \mathrm{~km} \cdot \mathrm{kbps}$ (e.g., a max theoretical data rate of $20 \mathrm{kbps}$ at a range of $2 \mathrm{~km}$ ) (Partan, 2006). Therefore, any type of multi-vehicle SLAM framework must adhere to the physical bandwidth limitations of the underwater domain.

In previous work, Eustice et al (2006c, 2007) developed a synchronous clock acoustic modem-based navigation system capable of supporting multi-vehicle ranging. The system consisted of a WHOI MicroModem (Freitag, 2005a, 2005b) (an underwater acoustic modem developed by Woods Hole Oceanographic Institution (WHOI)) and a low-power stable clock board. This system can be used as a 
synchronous-transmission communication/navigation system wherein data packets encode time of origin information as well as local ephemeris data (e.g., $x, y, z$ positional data and error metric). This allows for the direct measurement of inter-vehicle one-way travel time (OWTT) time-of-flight ranging. The advantage of an OWTT ranging methodology is that all passively receiving nodes within listening range are able to decode and measure the inter-vehicle range to the broadcasting node.

PeRL is currently investigating probabilistic fusion methods for a OWTT cooperative navigation multivehicle framework that scales across a distributed network of nodes in a non-fully-connected network topology. The proposed acoustic-modem navigation framework will exploit inter-vehicle OWTT ranging to supplement perceptual SLAM localization thereby reducing the need for state communication. The goal is to distribute state estimation between the vehicles in a coordinated fashion - allowing for navigation impoverished vehicles (e.g., no INS or DVL) to share from positional accuracies of better equipped vehicles (e.g., those with DVL bottom-lock, or VAN navigated vehicles near the seafloor).
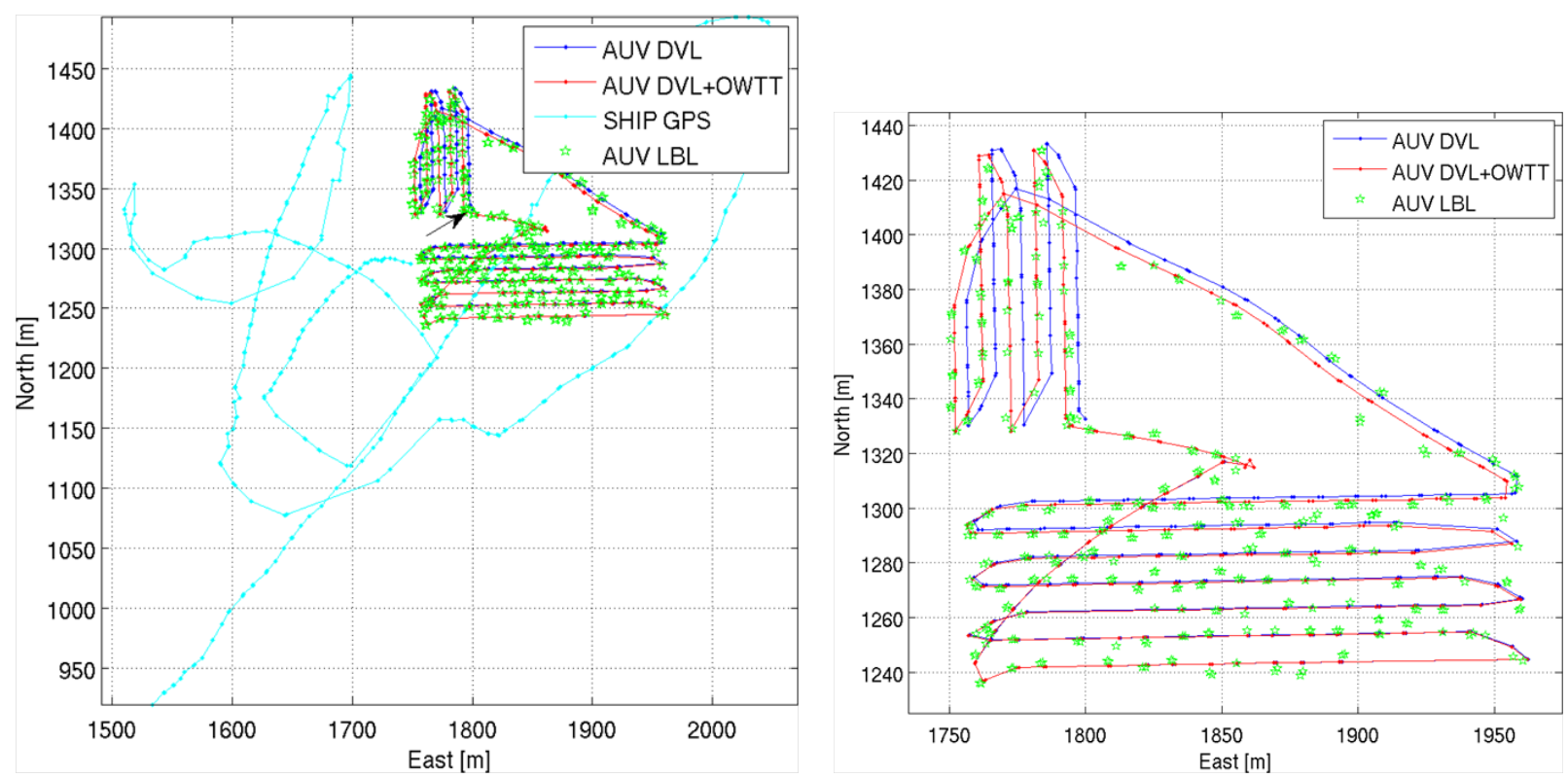

Figure 4. Preliminary OWTT results as reported in (Eustice, 2007) for a two node network consisting of an AUV and a surface ship. (left) Shown in blue is the raw dead-reckoned AUV trajectory; in cyan is the GPS-derived ship position; in red is the OWTT fused AUV trajectory; and in green is the LBL measured AUV position, which serves as an independent ground-truth. (right) Zoomed view of the AUV trajectory. 
Figure 4 depicts preliminary results, reported in (Eustice, 2007), demonstrating the OWTT proof of concept. Here, a GPS equipped surface ship navigationally aided a submerged AUV by broadcasting ship GPS position to the network while the AUV measured its range to the ship via the OWTTs.

\subsubsection{Perception-Driven Control}

Another research focus is in the domain of perception driven control. Algorithms are under development to enable a vehicle to respond to the environment by autonomously selecting alternative search patterns based upon perceived feature distributions in the environment. This creates improvements in survey efficiency by limiting duration in benign feature-poor areas, and instead spends more bottom-time over actual targets. A seafloor survey vehicle, for example, may drive into an area devoid of features during a mission. Instead of continuing to search the featureless space, where there is little return on investment from a visual navigation system, the vehicle would return to a previously known feature rich area and begin searching in another direction. PeRL is currently working on algorithms to assist in the decision making process of when to revisit known landmarks versus continuing new exploration. For example, Figure 5 depicts imagery from a hull inspection survey of a decommissioned aircraft carrier (Kim, 2009). At the one extreme we see that some areas of the hull are very texture-rich (heavily bio-fouled), while other areas are nearly feature-less (no distinguishing features such as weld seams, rivets, port opening, etc). In this type of environment, it makes no sense to incorporate imagery from the featureless region into the visual SLAM map because the image registration algorithm will fail to localize the vehicle due to a lack of visual features. Instead, by coupling the visual navigation feedback into the trajectory planning and control, the AUV can more intelligently adapt its survey and map building strategy so as to only return to feature-rich areas of the hull when it accrues too much pose uncertainty. By jointly considering along-hull pose uncertainty, map feature content, and energy expenditure accrued during AUV transit, we can frame the online path planning 
problem as a multi-objective optimization problem that seeks to find an optimal trajectory with respect to the possibly divergent criteria of exploration versus localization.

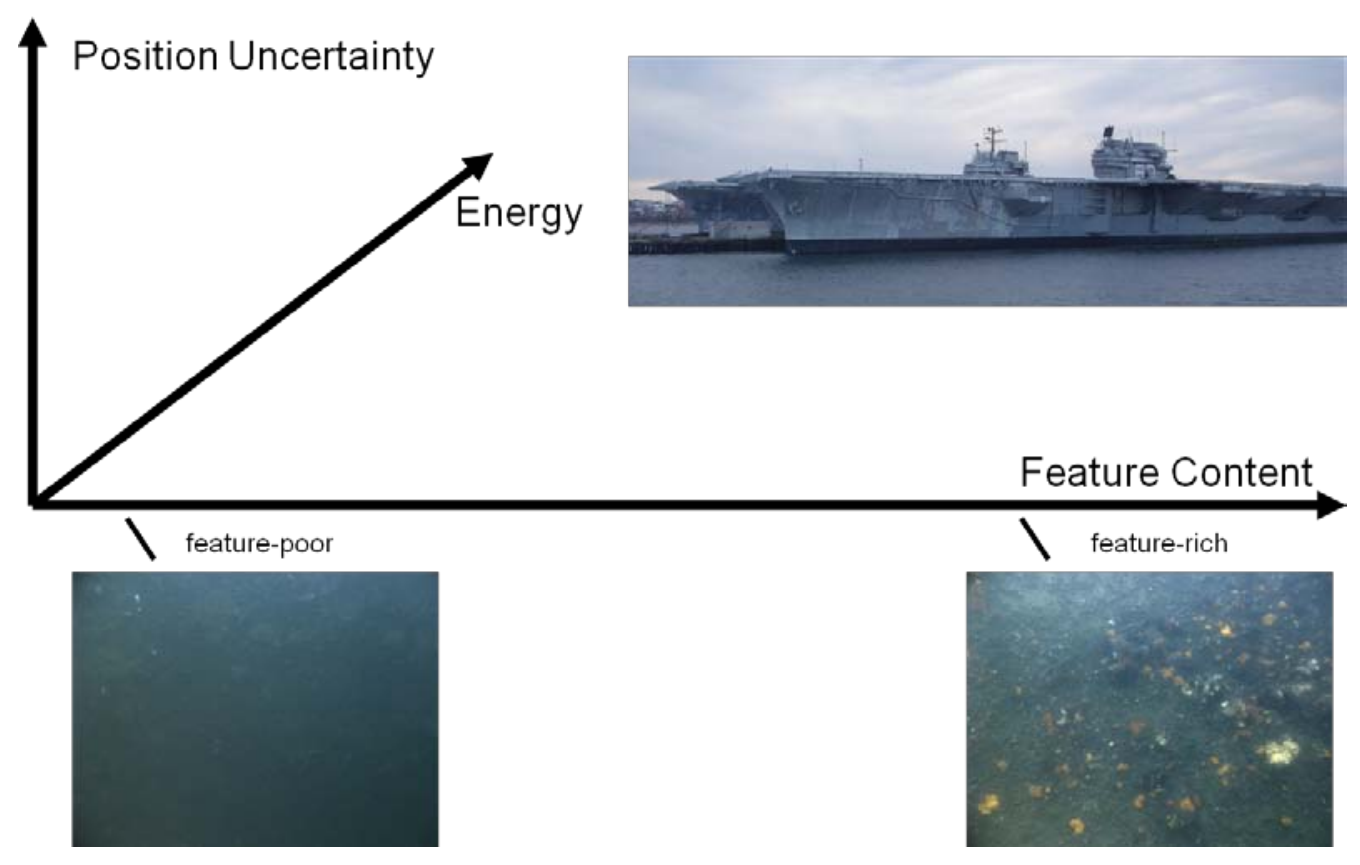

Figure 5. Depiction of the multi-objective constraints at play in perception driven control.

\section{Overview of PeRL's AUV Testbed}

To pursue PeRL's three research thrust areas, two commercial Ocean-Server Iver2 AUV systems were purchased and modified to serve as a real-world testbed platform for SLAM research at UMich. Although several other vehicle platforms currently include stereo-vision systems and DVL sensors, the Iver2 (Figure 6) was selected as a testbed development platform due to its ability to be transported in a personal vehicle and launched by a single user. The vehicles, as shipped, are rated to a depth of $100 \mathrm{~m}$, have a maximum survey speed of approximately $2 \mathrm{~m} / \mathrm{s}$ (4 knots), and weigh approximately $30 \mathrm{~kg}$ allowing for transport by two people (Anderson, 2005). 

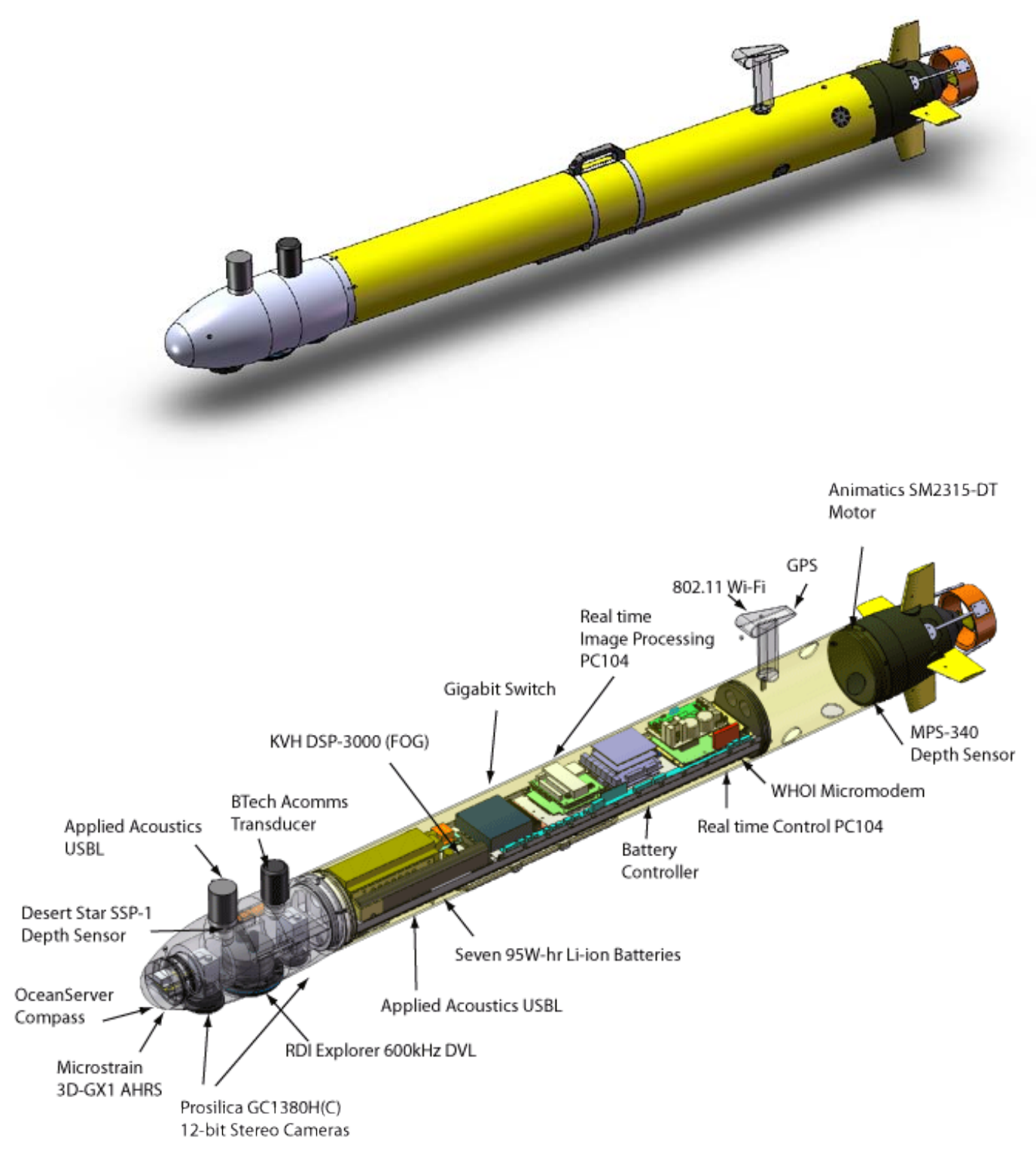

Figure 6. PeRL's modified Ocean-Server Iver2: external amd internal view.

Since the COTS (commercial-off-the-shelf) Iver2 vehicle does not come equipped with camera or DVL sensing, sensor upgrades were required to enable the stock vehicle to perform SLAM and coordinated multi-AUV missions. PeRL upgraded the vehicles with additional navigation and perception sensors (detailed in Figure 6b and Table 1) including 12-bit stereo down-looking Prosilica cameras, a Teledyne RDI 600 kHz Explorer DVL, a KVH single-axis fiber optic gyroscope (FOG), a Microstrain 3DM-GX1 attitude-heading-reference sensor, a Desert Star SSP-1 digital pressure sensor, and a WHOI Micro- 
modem for inter-vehicle communication. For illumination, we contracted the custom design and fabrication of a LED array by Farr and Hammar Engineering Services, LLC. To accommodate the additional sensor payload, a new Delrin nose cone was designed and fabricated. Additional 32-bit embedded PC104 CPU hardware was added for data-logging, real-time control, and in situ real-time SLAM algorithm testing and validation. Details of the design modification are discussed herein.

TABLE 1: INTEGRATED SENSORS ON THE PERL VEHICLES.

\begin{tabular}{|c|c|c|c|c|c|}
\hline IVER2 INSTRUMENTS & VARIABLE & UPDATE RATE & PRECISION & RANGE & DRIFT \\
\hline $\begin{array}{l}\text { Ocean-Server OS5000 } \\
\text { Compass }\end{array}$ & Attitude & $0.01-40 \mathrm{~Hz}$ & $1-3^{\circ}$ (Hdg), $2^{\circ}$ (Roll/Pitch) & $360^{\circ}$ & - \\
\hline $\begin{array}{l}\text { Measurement Specialties } \\
\text { Pressure Sensor MSP-340 }\end{array}$ & Depth & $0.01-40 \mathrm{~Hz}$ & $<1 \%$ of FS ${ }^{\dagger}$ & $0-20.4 \mathrm{~atm}$ & - \\
\hline $\begin{array}{l}\text { Imagenex Sidescan Sonar } \\
\text { (Dual Freq.) }\end{array}$ & Sonar image & 330 or $800 \mathrm{kHz}$ & - & $15-120 \mathrm{~m}$ & - \\
\hline USGlobalSat EM-406a GPS & XYZ position & $1 \mathrm{~Hz}$ & $5-10 \mathrm{~m}$ & - & - \\
\hline NEW INSTRUMENTS & & & & & \\
\hline $\begin{array}{l}\text { Prosilica GC1380H(C) Camera } \\
\text { (down-looking stereo-pair) }\end{array}$ & $\begin{array}{l}\text { Gray/color } \\
\text { image }\end{array}$ & $0.1-20 \mathrm{fps}$ & $\begin{array}{l}1360 \times 1024 \text { pixels } \\
12 \text {-bit depth }\end{array}$ & - & - \\
\hline Strobed LED Array* & Illumination & $0.1-4 \mathrm{fps}$ & - & 2-4 m altitude & - \\
\hline $\begin{array}{l}\text { Teledyne RDI } 600 \text { kHz } \\
\text { Explorer DVL }\end{array}$ & Body velocity & $7 \mathrm{~Hz}$ & 1.2-6 cm/s (@1 m/s) & $0.7-65 \mathrm{~m}$ & - \\
\hline KVH DSP-3000 1-Axis FOG & Yaw rate & $100 \mathrm{~Hz}$ & $1-6^{\circ} / \mathrm{h}$ & $\pm 375^{\circ} / \mathrm{s}$ & $4^{\circ} / \mathrm{h} / \mathrm{VHz}$ \\
\hline $\begin{array}{l}\text { Desert-Star SSP-1 300PSIG } \\
\text { Digital Pressure Transducer }\end{array}$ & Depth & $0.0625-4 \mathrm{~Hz}$ & $0.2 \%$ of FSt & $0-20.4 \mathrm{~atm}$ & - \\
\hline Applied Acoustics USBL & $\mathrm{XYZ}$ position & $1-10 \mathrm{~Hz}$ & \pm 0.1 m (Slant Range) & $1000 \mathrm{~m}$ & - \\
\hline OWTT $\ddagger$ Nav (Modem+PPS) & Slant range & Varies & 18.75 cm (@1500 m/s) & Varies & $<1.5 \mathrm{~m} / 14 \mathrm{~h}$ \\
\hline •WHOI Micro-modem & Communication & Varies & - & Varies & - \\
\hline -Seascan SISMTB v4 PPS Clock & Time & $1 \mathrm{~Hz}$ & $1 \mu \mathrm{s}$ & - & $<1 \mathrm{~ms} / 14 \mathrm{~h}$ \\
\hline Microstrain 3DM-GX1 AHRS & $\begin{array}{l}\text { Attitude } \\
\text { Angular rate }\end{array}$ & $\begin{array}{l}1-100 \mathrm{~Hz} \\
1-100 \mathrm{~Hz}\end{array}$ & $\begin{array}{l}2^{\circ}(\mathrm{Hdg}), 2^{\circ} \text { (Roll/Pitch) } \\
3.5^{\circ} / \mathrm{s}\end{array}$ & $\begin{array}{l}360^{\circ} \\
\pm 300^{\circ} / \mathrm{s}\end{array}$ & $-\overline{210^{\circ} / \mathrm{h} / \mathrm{vHz}}$ \\
\hline
\end{tabular}

\subsection{Mechanical/Electrical Design and Integration}


The design goals during the integration phase of vehicle development consisted of minimizing hydrodynamic drag, maintaining neutral buoyancy, and maximizing sensor payload capacity within the pressure hull. These requirements were achieved through the use of lightweight materials such as acrylonitrile butadiene styrene (ABS), Delrin, and aluminum, and careful center of buoyancy and center of mass computations. The entire vehicle was modeled using SolidWorks solid modeling software and extensive use of these computer aided design (CAD) models provided optimal arrangements of internal components prior to actual installation (Figure 7).

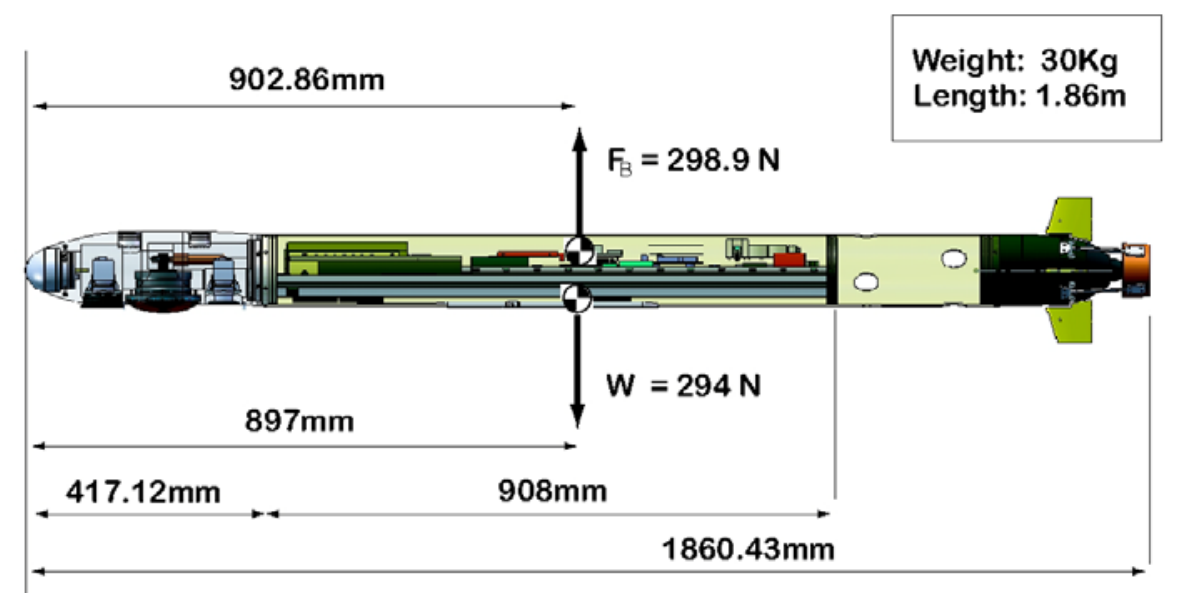

Figure 7. Mechanical layout.

The addition of a redesigned SLAM nose cone and sensor payload shifted both the original center of buoyancy and center of gravity. New positions were estimated using the CAD models and refined during ballast tests at the UMich Marine Hydrodynamics Laboratory (MHL). The vehicle is ballasted to achieve approximately $0.11 \mathrm{~kg}(0.25 \mathrm{lbs})$ reserve buoyancy for emergency situations when the vehicle must surface without power. Vehicle trim is set neutral to achieve passive stability and to optimize both diving and surfacing operations.

The interior component arrangement within the main body took into account mass, geometry, heat dissipation, and electrical interference considerations when determining the spatial layout and 
arrangement of sensors, computing, and electronics in the main tube. Due to the high density of sensors and other devices in the pressure housing, the components with the highest heat radiation, such as computers and DC-DC converters, were placed in direct contact with the aluminum chassis to allow better heat dissipation. Also, sensors that are prone to electrical noise from surrounding electronics were spatially separated in the layout (e.g. the MEMS Microstrain 3DM-GX1 is located in the nose cone tip, the furthest point from motor and battery pack influence).

Electrically, the vehicle is powered by a $665 \mathrm{~W} \cdot \mathrm{hr}$ Li-ion battery pack made up of seven $95 \mathrm{~W} \cdot \mathrm{hr}$ laptop batteries. This battery pack is managed by an Ocean-Server Intelligent Battery and Power System module. The additional sensors and PC104 computing added by PeRL draw less than $40 \mathrm{~W}$ total. This load is in addition to the original $12 \mathrm{~W}$ nominal vehicle hotel load and $110 \mathrm{~W}$ propulsion load of the stock Iver2, which results in a combined maximum total power draw of approximately $162 \mathrm{~W}$ (this assumes full hotel load and the motor at full power). The estimated run time at full load is 4.1 hours; however, taking into account a more realistic assessment of propulsion load for photo transect survey conditions, the continuous run time will be closer to $5.2+$ hours at a 2 knot (i.e., $75 \mathrm{~W}$ propulsion) survey speed.

\subsection{SLAM Nose Cone}

In order to support the real-time visual SLAM objectives of PeRL, a down-looking stereo-vision system was added to the Iver2 vehicles. A dual camera arrangement was chosen because it provides greater flexibility for visual-SLAM research. For example, using a stereo-rig allows for the measurement of metrical scale information during pairwise image registration and, thereby, can be used to improve odometry estimation by observing velocity scale error in DVL bottom-track measurements. Additionally, the dual camera arrangement can be used to run one of the cameras in a low-resolution mode suitable for real-time image processing (e.g. VGA or lower), while the second camera can be run at its native 1.6 megapixel resolution for offline processing. At depth, illumination will come from a LED array mounted 
at the aft of the vehicle. The array will provide optimal illumination over a 2-4 m imaging altitude at up to $4 \mathrm{fps}$ strobe rate with a camera-to-light separation distance of approximately $1.25 \mathrm{~m}$.

To accommodate the two-camera vision system and the DVL transducer, a new nose cone was designed and fabricated. The UMich custom-designed nose cone (Figure 8) was fabricated from Acetron GP (Delrin) due to the material's high tensile strength, scratch resistance, fatigue endurance, low friction, and low water absorption. Threaded inserts are installed in the nose cone to prevent stripped threads and stainless fasteners with a polytetrafluoroethene (PTFE) paste (to prevent corrosion issues) are used in all locations.

The designed working depth of the nose cone is $100 \mathrm{~m}$ (to match the full rating of the Iver2). Calculations were performed according to ASME Section VIII Boiler and Pressure Vessel Code to verify wall thickness in each of the nose cone sections. A minimum factor of safety of at least 2.64 was attained for all sections of the nose cone. Pressure tests, conducted at Woods Hole Oceanographic Institution, demonstrated the structural integrity of the nose cone to $240 \mathrm{~m}$ water depth. Three short duration tests of 12 minutes each were made to $24.5 \mathrm{~atm}$ ( $240 \mathrm{~m}$ salt water equivalent) and one long duration test of five hours was made to $24.5 \mathrm{~atm}$.

The Teledyne RDI $600 \mathrm{kHz}$ Explorer DVL is integrated into the nose cone using fasteners to attach the DVL head to threaded inserts in the nose cone material. The limited internal cavity space of the Iver2 nose precludes the use of RDI's recommended clamp attachment method. Instead, self-sealing fasteners are used to eliminate a fluid path through the mounting holes of the DVL to the interior of the nose cone. The associated DVL electronics module is mounted in the main chassis of the vehicle just behind the forward bulkhead. The electronics module and transducer head are connected by RDI's recommended shielded twisted-pair cabling to reduce stray electromagnetic field (EMF) effects. 
Two nose cone plugs were designed for camera integration. These plugs include a sapphire window and two mounting brackets each (Figure 8). The synthetic sapphire window was custom designed and fabricated by the Optarius Company of the U.K. Sapphire was chosen due to its high scratch resistance and superior tensile strength to that of plastic or glass materials. The mounting brackets were designed in CAD and printed in ABS plastic using a Dimension FDM Elite rapid prototype machine. Static face and edge o-ring seals prevent water ingress through the plug around the sapphire window.
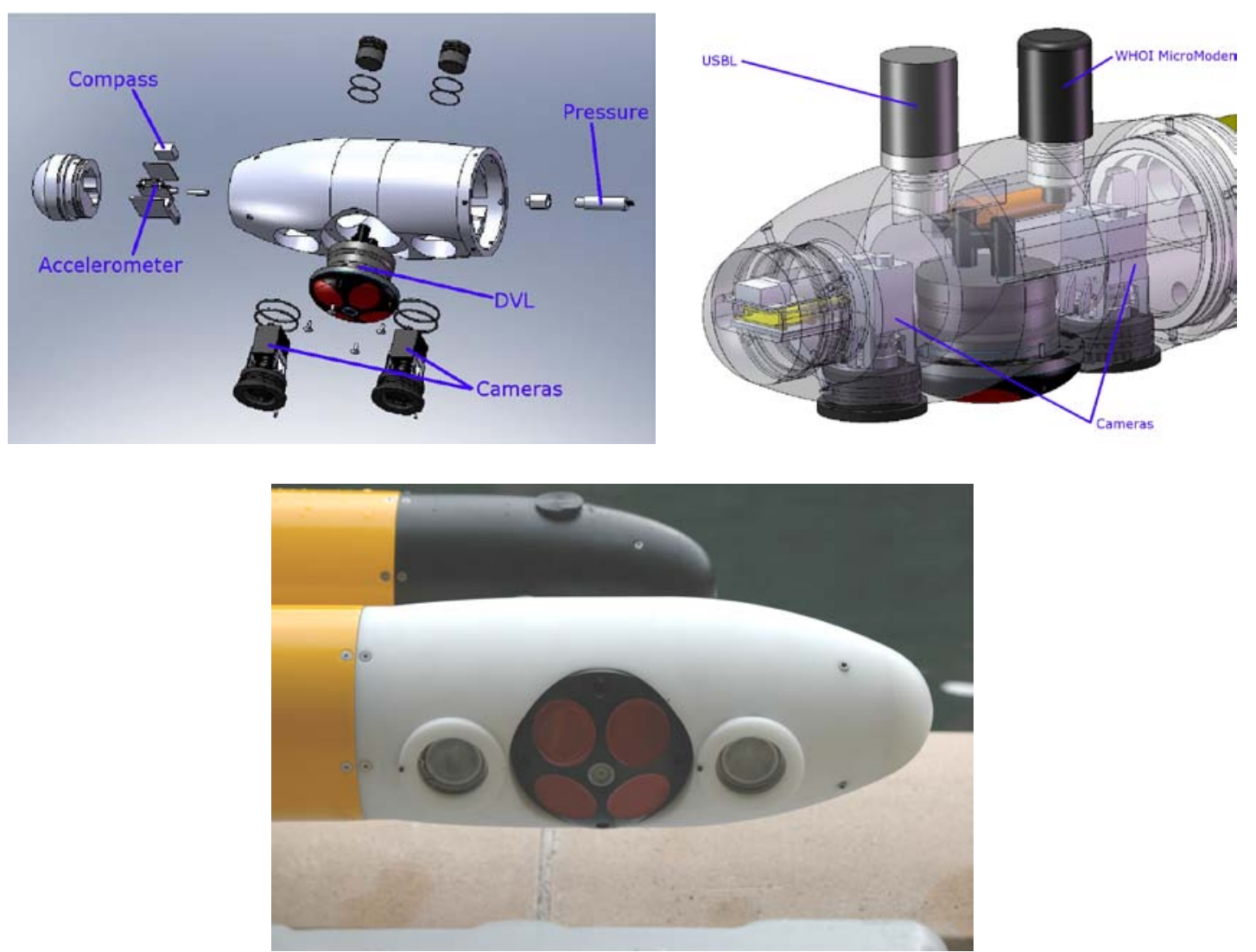

Figure 8. (top) Exploded and transparent view of PeRL's redesigned nose cone. (bottom) The fabricated nose cone as installed.

A Desert Star SSP-1 pressure transducer is mounted to an internal face of the nose cone and is exposed to the ambient environment through a $1 / 8^{\prime \prime}$ shaft drilled perpendicular to the nose cone wall to reduce flow noise influence on the sensor. The Microstrain 3DM-GX1 is integrated into the nose cone tip by mounting the Ocean-Server OS5000 compass on top of the 3DM-GX1 and milling a cavity in the tip to 
allow for the additional vertical clearance. All o-rings installed in the nose cone are of material Buna-N (acrylonitrile-butadiene) and are lightly lubricated with Dow Corning \#4 prior to installation.

\subsection{Mission Planning and Control}

The stock Iver2 control computer is a $500 \mathrm{MHz}$ AMD Geode CPU running Windows XP Embedded for the operating system. Ocean-Server provides a GUI mission planning module called VectorMap (Figure 11) and vehicle control software called UVC (Underwater Vehicle Control). The VectorMap mission planning software allows the user to graphically layout a mission trajectory over a geo-registered image or nautical navigation chart, and to specify parameters such a depth, speed, goal radius, timeout and other attributes for each waypoint. The output of VectorMap is an ASCII waypoint file that the UVC loads and executes within a PID control framework (Leveille, 2007). Additionally, the UVC supports a backseat driver API interface for user-level control from a host client. This API supports two control primitives: 1) a high-level control interface where the host specifies the reference heading, speed, and depth set points; and 2) a low-level control interface where the host specifies servo commands for the thruster and control surfaces.

The modified PeRL vehicle uses a Digital-Logic ADL945 1.2GHz Core-Duo PC104 for backseat control and data logging. The ADL945 runs less than $10 \mathrm{~W}$ in power consumption, has Gigabit Ethernet for interfacing with the Prosilica GigE cameras, and runs 32-bit Ubuntu Linux. The host stack records data from the cameras and navigation sensors, performs online state estimation, and directs the real-time control of the Iver2 via the backseat driver API. For ease of software development and modularity we have adopted a multi-process software paradigm that uses the open-source LCM inter-process communication library developed by the MIT DARPA Urban Grand Challenge team (Leonard, 2008; LCM, 2009).

\section{MISSIONS AND TESTING}


Current missions and testing conducted by PeRL include testing at the UMich Marine Hydrodynamics Laboratory tow tank, automated visual ship hull inspection (conducted at AUVFest'08), field testing at the University of Michigan Biological Station, and archaeological surveys of shipwrecks in the Thunder Bay National Marine Sanctuary.

\subsection{University of Michigan Marine Hydrodynamics Laboratory}

The UMich Marine Hydrodynamics Laboratory (MHL) provides a controlled experimental environment for testing real-time underwater visual SLAM algorithms. The freshwater physical model basin measures $110 \mathrm{~m} \times 6.7 \mathrm{~m} \times 3.0 \mathrm{~m}$ (Figure 9), and can achieve prescribed vehicle motions via the electronically controlled tank carriage. Trajectory ground-truth is obtained from encoder measured carriage position and will be used to validate real-time visual SLAM pose estimates derived from registering imagery of the tank floor. For ease of real-time algorithm development, we can attach a wet-mateable wired ethernet connection to a vehicle bulkhead so that imagery and sensor data can be streamed live to a topside desktop computer. This allows for greater flexibility in real-time software development, visualization, and debugging. Use of the test facility has been beneficial for early development and testing of our Iver2 hardware and real-time underwater visual SLAM algorithms.
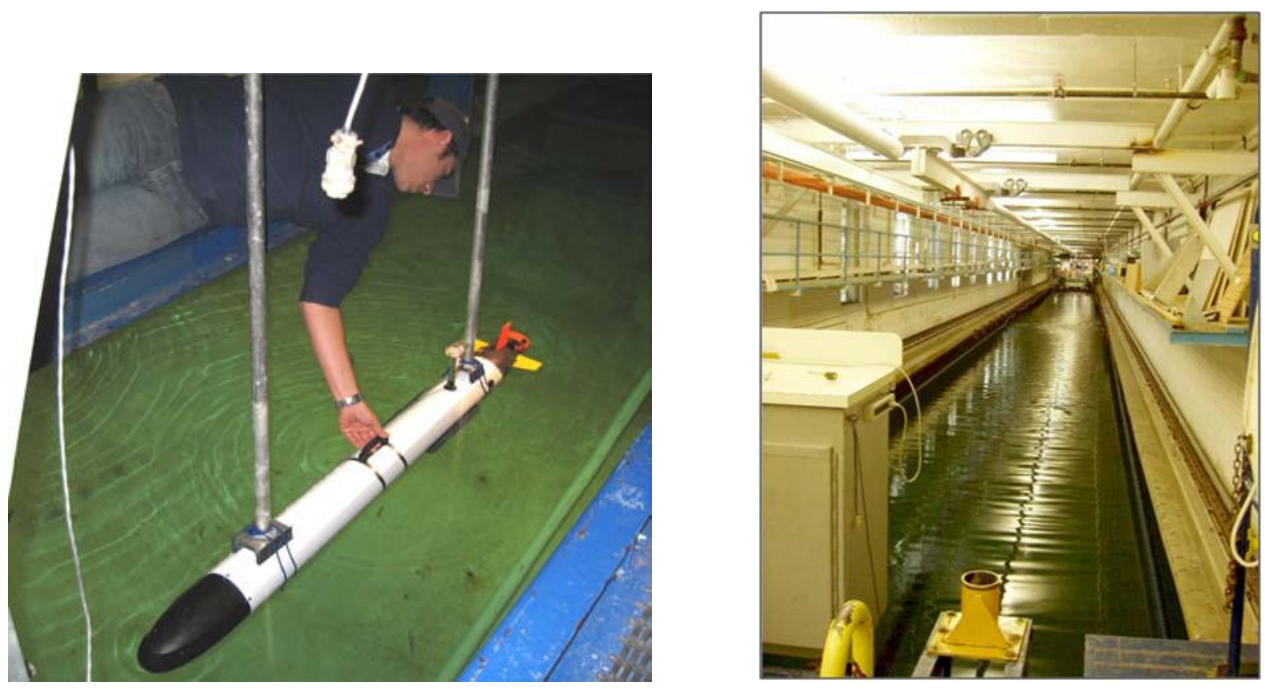

Figure 9. Vehicle testing at MHL tow tank. 


\subsection{AUVFest'08}

The Iver2 serves as a testbed for real-time visual autonomous port and hull inspection algorithm research at UMich. We use the Iver2 as a proxy for visual hull inspection by developing and testing our algorithms to navigate over the seafloor (since the underlying visual navigation algorithm is fundamentally the same in the two scenarios). This allows us to use the Iver2 to validate the accuracy of our visually augmented navigation method, and to test and implement our real-time algorithms on the type of embedded system hardware typically found on AUVs.

Meanwhile, to test our VAN algorithms in a real hull-inspection scenario, PeRL collaborated with Massachusetts Institute of Technology (MIT) and Bluefin Robotics at AUVFest'08 to put one of our camera systems on the Hovering Autonomous Underwater Vehicle (HAUV) (Vaganay, 2005). In this experiment, we collected imagery of the hull of the USS Saratoga - a decommissioned U.S. aircraft carrier stationed at Newport, Rhode Island (Figure 10a). PeRL packaged and mounted a calibrated Prosilica GC1380HC camera (the same camera system as used in the Iver2 SLAM nose cone) and flash strobe light system on the HAUV hull inspection vehicle. Boustrophedon survey imagery was collected by the HAUV of the hull of the USS Saratoga. The HAUV is equipped with a $1200 \mathrm{kHz}$ DVL, fiber optic gyro (FOG), and depth sensor, all of which are comparable to the sensor suite integrated into PeRL's Iver2 testbed.

Preliminary results for visual hull-relative navigation are shown in Figure 10 (Kim, 2009). Here we see a pose-graph of the camera constraints generated through pairwise registration of overlapping imagery. These constraints are fused with navigation data in an extended information filter framework to provide bounded error precision navigation along the hull. Each node in the network corresponds to a digitalstill image taken along the hull (over 1300 images in all). Note the discrete dropout of imagery along the second leg in the region of $5 \mathrm{~m}$ to $20 \mathrm{~m}$ along the hull axis. Due to a logging error, we did not record any imagery during this time period; however, this gap in the visual data record actually highlights the utility 
of our hull-referenced visual navigation approach. Because we are able to pairwise register views of the hull taken from different times and locations, the camera-based navigation algorithm is able to "closethe-loop" and register itself to earlier imagery from the first leg of the survey - thereby resetting any incurred DVL navigation error during the data dropout period. It is precisely this hull-referenced navigation capability that allows the AUV to navigate in situ along the hull without the need for deploying any external aiding (e.g., acoustic beacon transponders).
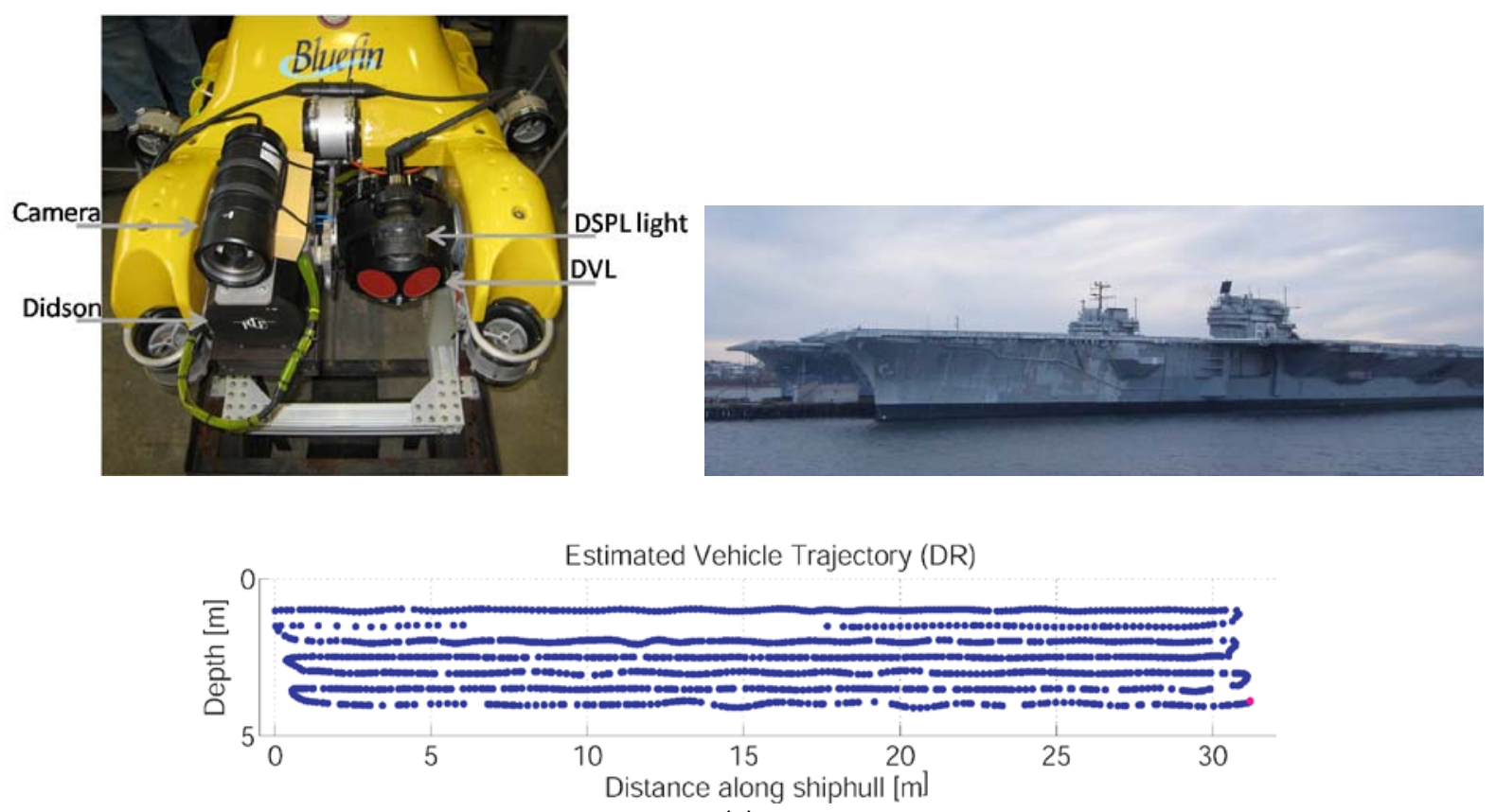

(a)

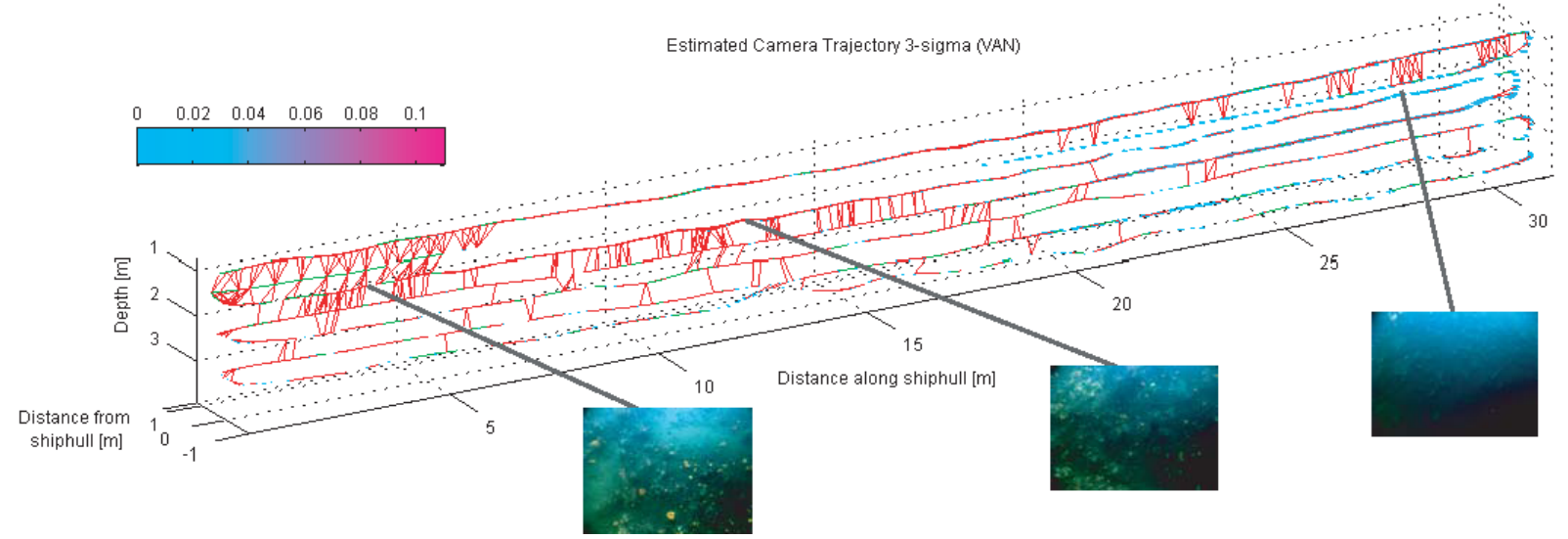

(b) 


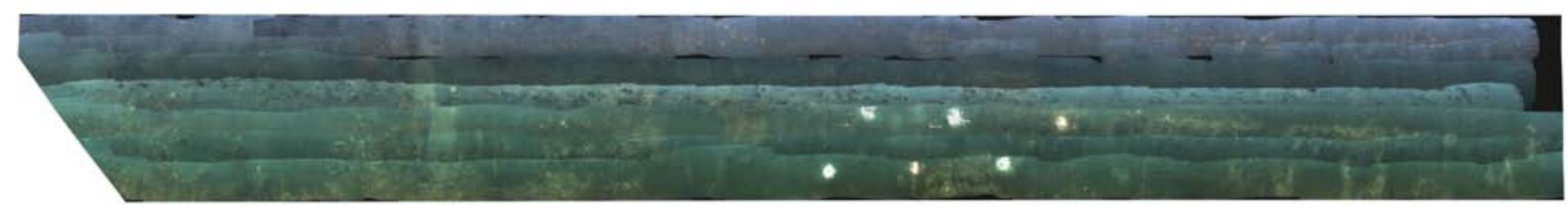

(c)

Figure 10. Hull inspection results from AUVFest'08. (a) Depiction of the AUVFest'08 experimental setup. (b) The cameraderived pose constraints are shown as red and green links. Each vertex represents a node in the pose-graph enclosed by its $3 \sigma$ covariance uncertainty ellipsoid. Due to the change of the visual feature richness along the hull, the uncertainty ellipsoid inflates when the vehicle is not able to build enough pose constraints, but then deflates once VAN creates camera constraints with previous tracklines. Three small figure insets depict the typical feature richness for different regions of the hull. (c) Triangulated 3D points are fitted to obtain a smooth surface reconstruction and then texture mapped to create a 3D photomosaic. The six white dots are targets that were manually placed on the hull and used to verify the utility of the visual hull inspection algorithm.

\subsection{University of Michigan Biological Station}

Field trials were held on Douglas Lake at the University of Michigan Biological Station (UMBS) in Pellston, Michigan during July, 2008. Four days of on-water testing demonstrated maneuverability, vehicle speed, dead-reckon navigation, wireless ethernet communication, sidescan sonar functionality, digital compass, and manual surface joystick operation modes as summarized in Table 2.

TABLE 2. RESULTS OF TESTING STOCK IVER2 AT UMBS.

\begin{tabular}{|c|c|}
\hline Attribute & Performance \\
\hline Maneuverability & $\begin{array}{l}1.5 \mathrm{~m} \text { altitude bottom following } \\
25 \mathrm{~cm} \text { depth band control } \\
4.5 \mathrm{~m} \text { turning radius }\end{array}$ \\
\hline Speed & $0.5-1.5 \mathrm{~m} / \mathrm{s}$ \\
\hline $\begin{array}{l}\text { DR navigation accuracy } \\
\text { (DR = prop counts }+ \text { compass } u / w \\
\text { GPS when on surface) }\end{array}$ & $10 \%$ distance traveled \\
\hline 802.11g Wi-Fi & $100 \mathrm{~m}$ range (on surface) \\
\hline
\end{tabular}

Launch and recovery were conducted from shore, dock and from a pontoon boat. A full sidescan sonar survey of the south-eastern bay at Douglas Lake was run from the UMBS docks (Figure 11). After completion of the mission, the vehicle was manually driven under joy-stick control from a portable wireless station back to the dock for recovery. This onshore launch and recovery capability facilitates ease of experimental field testing with the Iver2. 


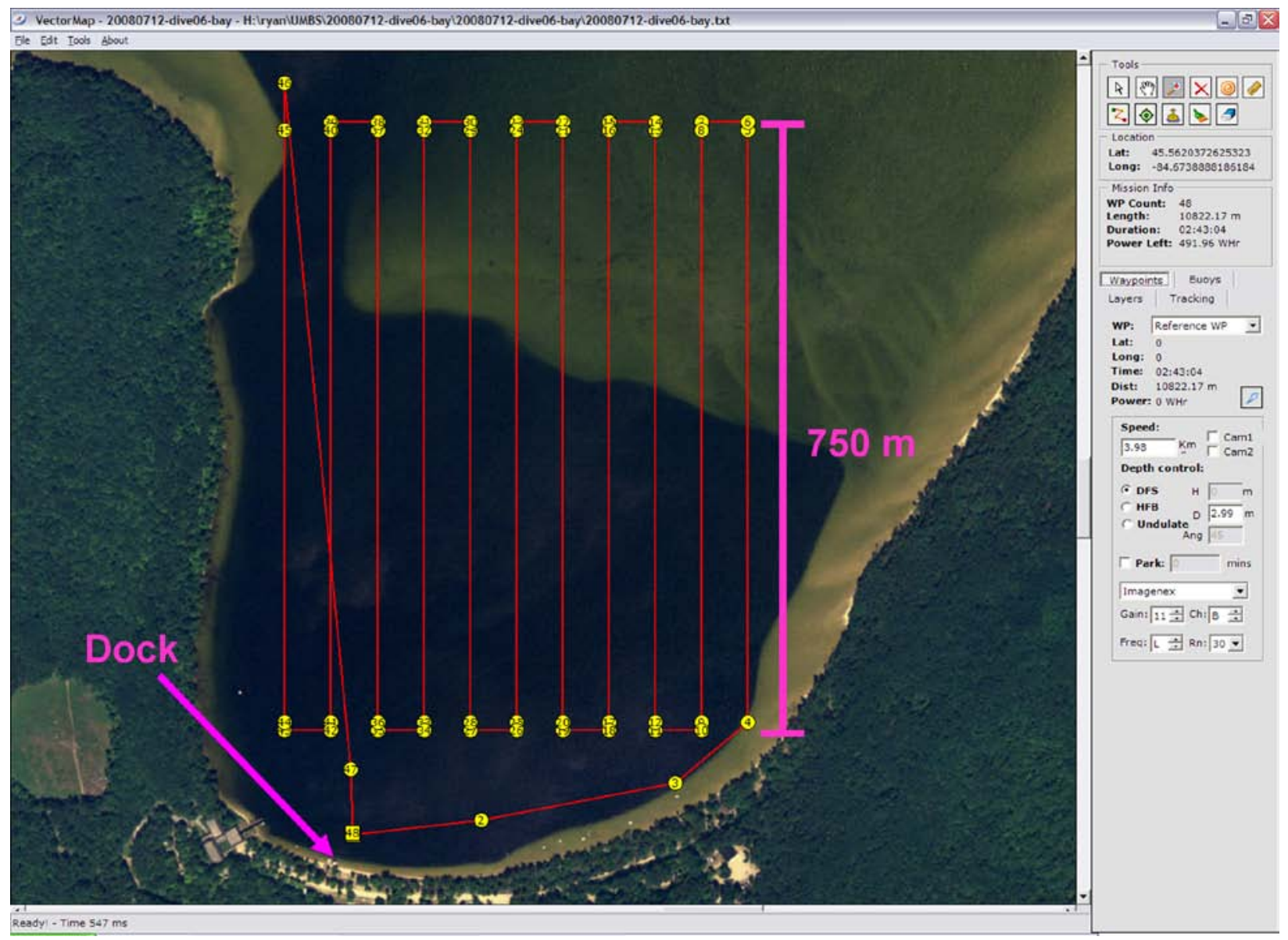

Figure 11. VectorMap mission-planning interface for the Iver2. The depicted tracklines are for a sidescan sonar survey of a portion of Douglas Lake. Launch and recovery of the Iver2 was performed from a dock on shore.

\subsection{Thunder Bay National Marine Sanctuary}

In August, 2008, PeRL collaborated with National Oceanic and Atmospheric Administration (NOAA)

Thunder Bay National Marine Sanctuary (TBNMS) researchers to map unexplored areas outside the Sanctuary's current boundaries (Figure 12a). Established in 2000, the TBNMS protects one of the nation's most historically significant collections of shipwrecks. Located in the northeast corner of Michigan's Lower Peninsula, the 448 square-mile sanctuary contains 40 known historic shipwrecks. Archival research indicates that over 100 sites await discovery within and just beyond the sanctuary's current boundaries. This fact, coupled with strong public support and the occurrence of dozens of known shipwrecks, provide the rationale for the sanctuary's desire to expand from 448 square-miles to 3,662 square-miles (an eight fold increase). To date, however, a comprehensive remote sensing survey 
has not been conducted in the potential expansion area. Moreover, significant portions of the existing sanctuary have not been explored.

PeRL is engaged in a five-year collaboration effort with TBNMS to use the Sanctuary as a real-world engineering testbed for our AUV algorithms research. TBNMS provides in-kind support of ship time and facilities, and in return receives SLAM-derived archaeological data products ranging from 3D photomosaics of ship wrecks to sidescan sonar maps of the Sanctuary seafloor. In addition, PeRL is engaged in public outreach efforts in collaboration with TBNMS to educate the general public in the use and technology of underwater robotics. In development is an AUV technology display in their state-ofthe-art Great Lakes Maritime Heritage Center, (a 20,000 square foot building featuring a 100-seat theater, 9,000 square feet of exhibit space, and distance learning capabilities) that will consist of an Iver2 AUV hull, a multimedia kiosk, and maps and data products derived from PeRL's field testing in the Sanctuary.

This past August, as part of a NOAA Ocean Exploration grant, PeRL fielded one of its two Iver2 AUVs to collect sidescan sonar imagery in unmapped regions of the Sanctuary seafloor. Figure 12 shows survey tracklines and sonar imagery collected of a newly found wreck outside of the Sanctuary's boundaries in approximately $50 \mathrm{~m}$ of water depth. 

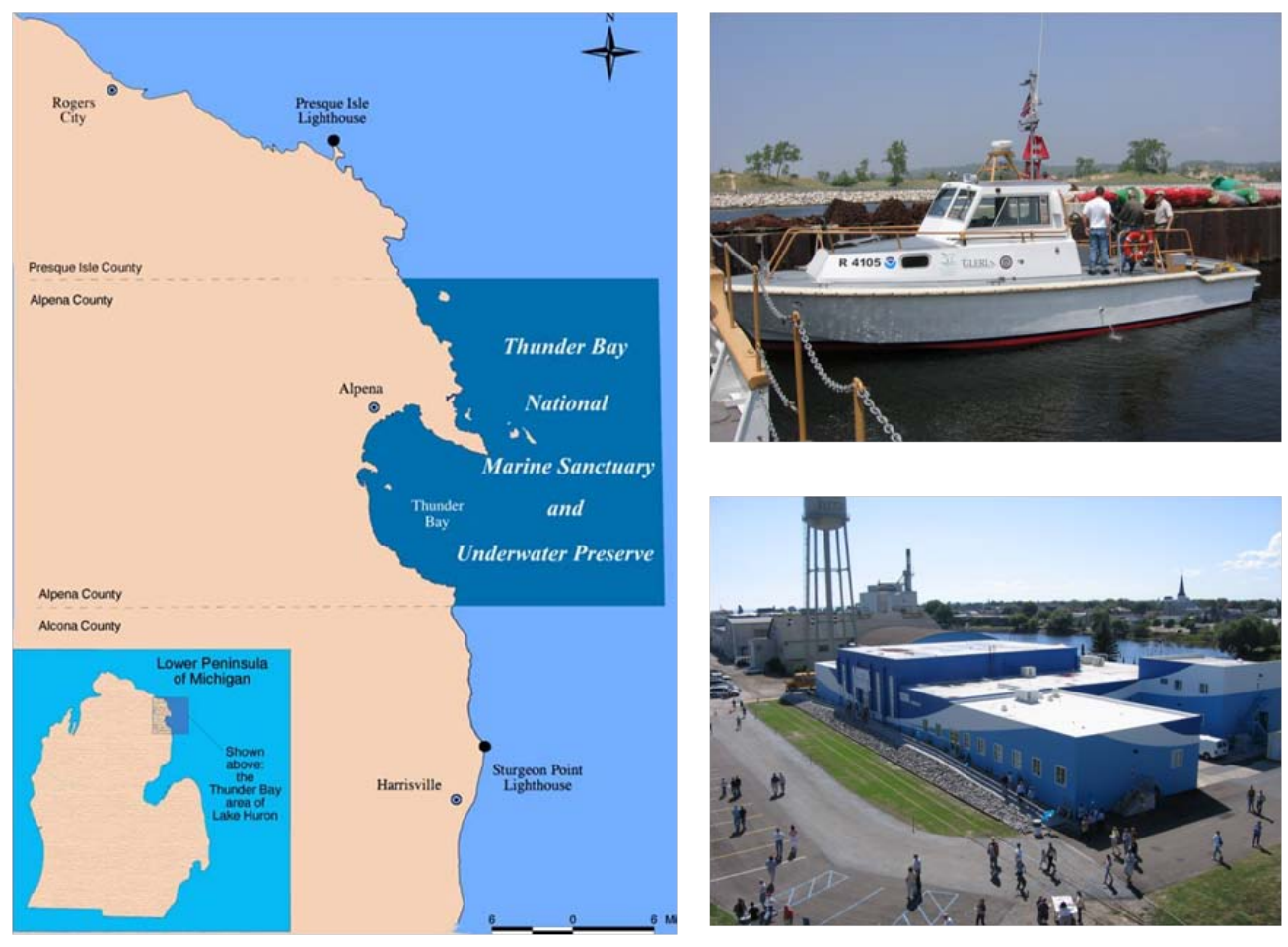

(a)

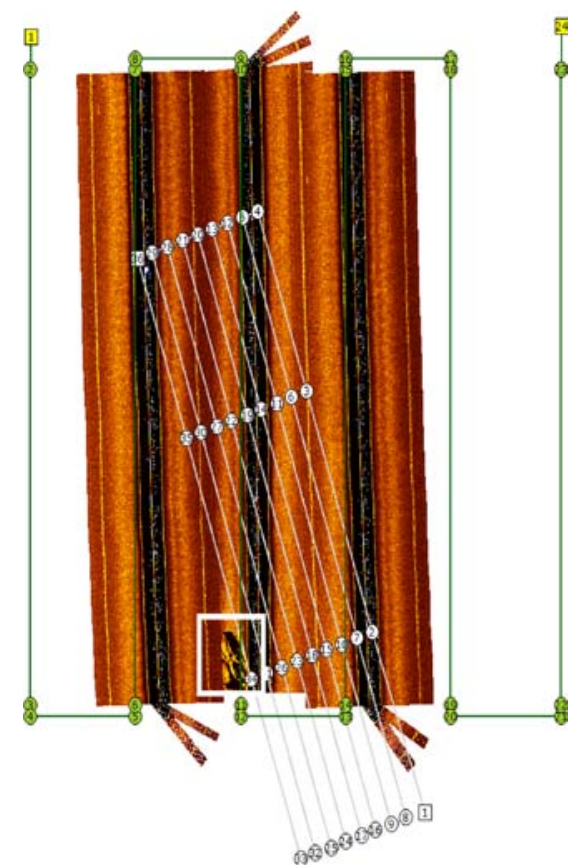

(b) Mission overlay.

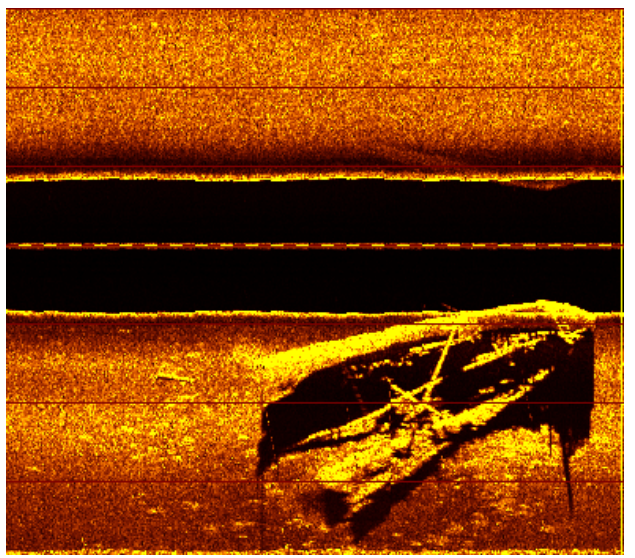

(c) $330 \mathrm{kHz}$ sidescan imagery.

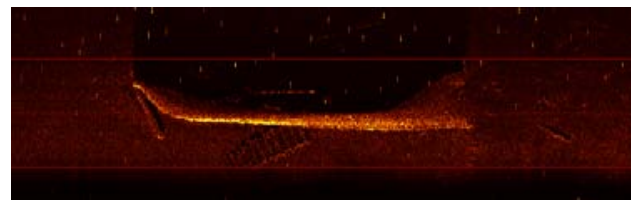

(d) $880 \mathrm{kHz}$ sidescan imagery

Figure 12. Sidescan sonar mapping results from the 2008 summer field season in TBNMS. (a) Thunder Bay National Marine Sanctuary facility. (b) A large area search was conducted first to locate the target wreck indicated in the white box (survey tracklines are shown in green). A second finer-scale survey was then conducted to map the target at higher resolution (tracklines overlaid in gray). (c) Target imagery found using $330 \mathrm{kHz}$ sonar. (d) Detailed target imagery using $800 \mathrm{kHz}$ sonar.

\section{CONCLUSION}


This paper provided an overview of PeRL's AUV algorithms research and testbed development at the University of Michigan. To summarize, PeRL's main research thrusts are in the areas of (1) real-time visual SLAM; (2) cooperative multi-vehicle navigation; and (3) perception-driven control. Toward that goal we reported the modifications involved in preparing two commercial Ocean-Server AUV systems for SLAM research at the University of Michigan. PeRL upgraded the vehicles with additional navigation and perceptual sensors including 12-bit stereo down-looking Prosilica cameras, a Teledyne RDI $600 \mathrm{kHz}$ Explorer DVL for 3-axis bottom-lock velocity measurements, a KVH single-axis fiber-optic gyroscope for yaw rate, and a WHOI Micro-modem for communication, along with other sensor packages. To accommodate the additional sensor payload, a new Delrin nose cone was designed and fabricated. Additional 32-bit embedded CPU hardware was added for data-logging, real-time control, and in situ real-time SLAM algorithm testing and validation. Our field testing and collaboration with the TBNMS will provide validation of the proposed navigation methodologies in a real-world engineering setting, while a new museum exhibit on underwater robotics at their visitor center will disseminate the findings and results of this research to the general public.

\section{ACKNOWLEDGMENTS}

This work is supported in part through grants from the National Science Foundation (Award \#IIS 0746455), the Office of Naval Research (Award \#N00014-07-1-0791), and a NOAA Ocean Exploration grant (Award \#WC133C08SE4089). 


\section{REFERENCES}

Anderson, B. and J. Crowell. 2005. Workhorse AUV - a cost-sensible new autonomous underwater vehicle for surveys/soundings, search \& rescue, and research. In: IEEE/MTS OCEANS Conference and Exhibition, pp. 1228-1233.

Bailey, T. and H. Durrant-Whyte. 2006. Simultaneous localization and mapping (SLAM): Part-II. IEEE Robotics and Automation Magazine. 13(3):108-117.

Ballard, R.D. (ed). 2008. Archaeological Oceanography. Princeton University Press. 296 pp.

Bourgault, F., T. Furukawa and H. Durrant-Whyte. 2004. Decentralized Bayesian negotiation for cooperative search. In: IEEE/RSJ International Conference on Intelligent Robots and Systems. pp. 26812686.

Durrant-Whyte, H. and T. Bailey. 2006. Simultaneous localization and mapping: Part-I. IEEE Robotics and Automation Magazine. 13(2):99-110.

Eustice, R.M., H. Singh and J.J. Leonard. 2006a. Exactly sparse delayed-state filters for view-based SLAM. IEEE Transactions on Robotics. 22(6):1100-1114.

Eustice, R.M., H. Singh, J.J. Leonard and M.R. Walter. 2006b. Visually mapping the RMS Titanic: Conservative covariance estimates for SLAM information filters. International J. Robotics Research. 25(12):1223-1242.

Eustice, R.M., L.L. Whitcomb, H. Singh and M. Grund. 2006c. Recent advances in synchronous-clock oneway-travel-time acoustic navigation. In: IEEE/MTS OCEANS Conference and Exhibition. pp. 1-6. Boston, MA.

Eustice, R.M., L.L. Whitcomb, H. Singh, and M. Grund. 2007. Experimental results in synchronous-clock one-way-travel-time acoustic navigation for autonomous underwater vehicles. In: IEEE International Conference on Robotics and Automation. pp. 4257-4264. Rome, Italy.

Eustice, R.M., O. Pizarro and H. Singh. 2008a. Visually augmented navigation for autonomous underwater vehicles. IEEE J. Oceanic Engineering. 33(2):103-122.

Eustice, R.M. 2008b. Toward real-time visually augmented navigation for autonomous search and inspection of ship hulls and port facilities. In: International Symposium on Technology and the Mine Problem. Monterey, CA.

Fleischer, S. 2000. Bounded-error vision-based navigation of autonomous underwater vehicles. PhD Thesis. Stanford University.

Foley, B., K. DellaPorta, D. Sakellariou, B. Bingham, R. Camilli, R. Eustice, D. Evagelistis, V. Ferrini, M. Hansson, K. Katsaros, D. Kourkoumelis, A. Mallios, P. Micha, D. Mindell, C. Roman, H. Singh, D. Switzer 
and T. Theodoulou. 2009. New methods for underwater archaeology: The 2005 Chios ancient shipwreck survey. J. Hesperia. In Print.

Freitag, L., M. Grund, S. Singh, J. Partan, P. Koski and K. Ball. 2005a. The WHOI micro-modem: an acoustic communications and navigation system for multiple platforms. In: IEEE/MTS OCEANS Conference and Exhibition. pp. 1086-1092. Washington, D.C.

Freitag, L., M. Grund, J. Partan, K. Ball, S. Singh and P. Koski. 2005b. Multi-band acoustic modem for the communications and navigation aid AUV. In: IEEE/MTS OCEANS Conference and Exhibition. pp. 10801085. Washington, D.C.

Hunt, M., W. Marquet, D. Moller, K. Peal, W. Smith and R. Spindel. 1974. An acoustic navigation system. Woods Hole Oceanographic Institution. Technical Report WHOI-74-6.

Kinsey, J.C., R.M. Eustice and L.L. Whitcomb. 2006. Underwater vehicle navigation: Recent advances and new challenges. In: IFAC Conference on Manoeurring and Control of Marine Craft. Lisbon, Portugal.

Kim, A. and R.M. Eustice. 2009. Pose-graph visual SLAM with geometric model selection for autonomous underwater ship hull inspection. In: IEEE/RSJ International Conference on Intelligent Robots and Systems. Submitted, Under Review.

LCM. 2009. Lightweight Communications and Marshalling. Retrieved April 15, 2009, from http://code.google.com/p/lcm/

Leonard, J., J. How, S. Teller, M. Berger, S. Campbell, G. Fiore, L. Fletcher, E. Frazzoli, A. Huang, S. Karaman, O. Koch, Y. Kuwata, D. Moore, E. Olson, S. Peters, J. Teo, R. Truax, M. Walter, D. Barrett, A. Epstein, K. Maheloni, K. Moyer, T. Jones, R. Buckley, M. Antone, R. Galejs, S. Krishnamurthy and J. Williams. 2008. A perception-driven autonomous urban vehicle. Journal of Field Robotics. 25(10):727774.

Leveille, E.A. 2007. Analysis, redesign and verification of the Iver2 autonomous underwater vehicle motion controller. Masters Thesis. University of Massachusetts Dartmouth.

Milne, P. 1983. Underwater acoustic positioning systems. Houston: Gulf Publishing Company. 284 pp.

Neira, J. and J. Tardos. 2001. Data association in stochastic mapping using the joint compatibility test. IEEE Transactions on Robotics and Automation. 17(6):890-897.

Newman, P.M. and J.J. Leonard. 2003. Pure range-only sub-sea SLAM. In: IEEE International Conference on Robotics and Automation. pp. 1921-1926.

Olson, E., J.J. Leonard and S. Teller. 2006. Robust range-only beacon localization. IEEE J. Oceanic Engineering. 31(4):949-958. 
Ong, L.L, B. Upcroft, T. Bailey, M. Ridley, S. Sukkarieh and H. Durrant-Whyte. 2006. A decentralised particle filtering algorithm for multi-target tracking across multiple flight vehicles. In: IEEE/RSJ International Conference on Intelligent Robots and Systems. pp. 4539-4544. Beijing, China.

Partan, J., J. Kurose and B. N. Levine. 2006. A survey of practical issues in underwater networks. In: ACM International Workshop on Underwater networks. pp. 17-24. New York: ACM Press.

Rekleitis, I., G. Dudek and E. Milios. 2003. Probabilistic cooperative localization and mapping in practice. In: IEEE International Conference on Robotics and Automation. pp. 1907-1912.

Ridley, M., E. Nettleton, S. Sukkarieh and H. Durrant-Whyte. 2002. Tracking in decentralised air-ground sensing networks. In: IEEE International Conference on Information Fusion. pp. 616-623.

Roman, C. and H. Singh. 2005. Improved vehicle based multibeam bathymetry using sub-maps and SLAM. In: IEEE/RSJ International Conference on Intelligent Robots and Systems. pp. 2422-2429.

Smith, S. and D. Kronen. 1997. Experimental results of an inexpensive short baseline acoustic positioning system for AUV navigation. In: IEEE/MTS OCEANS Conference and Exhibition. pp. 714-720.

Stewart, W. 1991. Remote-sensing issues for intelligent underwater systems. In: IEEE Conference on Computer Vision and Pattern Recognition. pp. 230-235.

Vaganay, J., M. Elkins, S. Willcox, F. Hover, R. Damus, S. Desset, J. Morash and V. Polidoro. 2005. Ship hull inspection by hull-relative navigation and control. In: IEEE/MTS OCEANS Conference and Exhibition. pp. 761-766.

Walter, M.R. and J.J. Leonard. 2004. An experimental investigation of cooperative SLAM. In: IFAC/EURON Symposium on Intelligent Autonomous Vehicles. Lisbon, Portugal.

Williams, S.B., G. Dissanayake and H. Durrant-Whyte. 2002. Towards multi-vehicle simultaneous localisation and mapping. In: IEEE International Conference on Robotics and Automation. pp. 2743-2748. Washington, DC. 